\title{
Perimetria azul-amarelo em usuários de tabaco-álcool
}

\author{
Blue-on-yellow perimetry in tobacco and alcohol consumers
}

\author{
José Fernando de Carvalho Júnior ${ }^{1}$ \\ Diana Danda 2 \\ Hellmann Dantas ${ }^{3}$ \\ Tatiana Azevedo Arraes ${ }^{4}$ \\ Elani Cavalcanti ${ }^{5}$
}

${ }^{1}$ Residente em Oftalmologia da Fundação Altino Ventura - Recife (PE) - Brasil.

${ }^{2}$ Residente em Oftalmologia da Fundação Altino Ventura

- Recife (PE) - Brasil.

${ }^{3}$ Oftalmologista do Hospital de Olhos de Pernambuco Recife (PE) - Brasil.

${ }^{4}$ Residente em Oftalmologia da Fundação Altino Ventura - Recife (PE) - Brasil.

5 Oftalmologista do Hospital de Olhos de Pernambuco Recife (PE) - Brasil.

Endereço para correspondência: Fundação Altino Ventura (FAV). Rua da Soledade, 170 - Recife (PE) CEP 50070-040

E-mail: fav@fundacaoaltinoventura.org.br

Recebido para publicação em 29.06.2005

Última versão recebida em 08.03.2006

Aprovação em 20.03.2006

\section{RESUMO}

Objetivo: Avaliar as alterações de campo visual em usuários crônicos de tabaco e álcool por meio da perimetria azul-amarelo estratégia 10-2. Métodos: Quarenta e dois olhos de vinte e um voluntários usuários de tabaco e álcool, todos do gênero masculino, foram selecionados após exame oftalmológico completo e normal, sendo submetidos a perimetria azul-amarelo estratégia 10-2. Quinze voluntários participaram do grupo controle. A análise dos dados foi realizada mediante gráfico da profundidade do defeito e número de pontos alterados. Resultados: Observouse que 40 olhos $(95,3 \%)$ dos usuários crônicos de tabaco e álcool, apresentaram maior freqüência de alterações no gráfico de profundidade do defeito $(>10 \mathrm{~dB})$ e 27 olhos $(64,3 \%)$ apresentaram número de pontos alterados ( $>10$ pontos $)$, $(\mathrm{p}<0,0001)$. Todos os voluntários que apresentaram alterações no gráfico de profundidade do defeito e número de pontos alterados apresentavam menos que $10 \mathrm{~dB}$ e 10 pontos alterados, respectivamente. Conclusão: Foram observados maiores números de pontos alterados e profundidade do defeito refletindo alterações nas células do sistema parvocelular, responsáveis pela função de cores nos pacientes usuários crônicos de tabaco-álcool, por meio da perimetria azul-amarelo 10-2.

Descritores: Alcoolismo; Tabagismo; Perimetria/métodos; Campos visuais

\section{INTRODUÇÃO}

O alcoolismo está entre os grandes problemas de saúde pública mundial. $\mathrm{O}$ abuso e dependência combinados afetam $8 \%$ da sociedade brasileira gerando grande custo social ${ }^{(1)}$, tornando-se uma das mais importantes entraves da saúde mental no Brasil ${ }^{(2)}$.

O consumo abusivo e malefícios do álcool já são amplamente estabelecidos em diversos sistemas ${ }^{(3)}$ e alguns estudos já estabeleceram importantes associações de alterações oftalmológicas com o abuso de tabaco e/ou álcool, iniciando-se durante o desenvolvimento, através do abuso durante a gestação, onde o etanol exerce seus efeitos mais pronunciados ${ }^{(4)}$.

As alterações campimétricas da neuropatia óptica nutricional álcool induzida por meio de campo visual convencional, estímulo branco sobre fundo branco, já foram estabelecidas ${ }^{(5)}$. No entanto, este exame não se tem mostrado eficaz em detectar outras doenças nos estágios iniciais, como no glaucoma ${ }^{(6)}$.

A perimetria de ondas curtas (azul-amarelo), capaz de identificar alterações funcionais três a cinco anos antes da perimetria convencional ${ }^{(7)}$, tem sido útil para detecção precoce de diversas entidades nosológicas, inclusive algumas desordens neuro-oftalmológicas ${ }^{(8)}$, mas em diversos trabalhos foram usadas amostras de usuários de tabaco-álcool em processo de reabilitação. 
O objetivo deste estudo foi avaliar as alterações de campo visual em usuários crônicos de tabaco e álcool por meio de perimetria azul-amarelo estratégia 10-2, visando investigar suas alterações.

\section{MÉTODOS}

O estudo, prospectivo, tipo caso-controle, foi realizado na Fundação Altino Ventura, no período de março a outubro de 2004.

Fez parte do grupo estudo 21 usuários de tabaco-álcool. No grupo controle foi incluído 15 indivíduos não usuários de tabaco-álcool. Todos os voluntários eram do gênero masculino. A média de idade dos usuários de tabaco e álcool foi de $46,8 \pm 9,0$ anos e a do grupo controle de 44,2 $\pm 7,0$ anos. Esses indivíduos foram submetidos a exame oftalmológico de acuidade visual com e sem correção, refração, biomicroscopia, tonometria de aplanação e fundoscopia.

Foram excluídos aqueles que apresentaram pressão intraocular (PIO) igual ou maior que $21 \mathrm{mmHg}$, acuidade visual pior que 1 , escavação papilar maior ou igual a 0,5 ou assimetria maior que 0,2 , catarata nuclear densa, capsular anterior maior ou igual a $3+$ e subcapsular posterior maior ou igual a $2+$,e quaisquer alterações coriorretinianas, bem como aqueles voluntários que apresentavam limitações para o entendimento e realização da campimetria.

Realizou-se a perimetria azul amarelo Humphrey modelo HFA II 750, teste central 10-2, estratégia limiar total, pelo mesmo examinador, após um período de adaptação em sala escura com luz amarela por 3 minutos. O primeiro exame foi considerado para o estudo, mas os que apresentaram índices de confiabilidade alterados, foram excluídos de ambos os grupos.

A análise dos dados foi realizada através do gráfico da profundidade do defeito e número de pontos alterados, uma vez que, neste teste não se dispõe de índices globais como o "glaucoma hemifield test" (GHT), os índices "mean deviation" (MD), "pattern standard deviation" (PSD), "short flutuation" (SF) e "correct pattern standard deviation" (CPSD).

Os resultados quantitativos foram expressos por suas médias e desvios-padrão. Os resultados qualitativos foram expressos por suas frequiências absoluta e relativa. Foi usado o teste "t" de Student para amostras não pareadas com o objetivo de verificar possíveis diferenças entre médias. Foi usado o teste exato de Fisher para analisar possíveis diferenças entre freqüências. Aceitou-se p<0,05 para rejeição de nulidade.

O projeto de investigação foi aprovado pelo comitê de ética da Fundação Altino Ventura. Os voluntários só foram incluídos na investigação após assinatura do termo de consentimento livre e esclarecido.

\section{RESULTADOS}

Todos os voluntários usuários ou não usuários de tabacoálcool, apresentaram ótimos índices de confiabilidade com respostas falsas positivas, falsas negativas e perda de fixação dentro dos limites aceitáveis.

O valor médio do tempo de uso de tabaco-álcool foi de 19,4 $\pm 5,6$ anos. A quantidade média de álcool ingerida em litros/dia foi de $1,90 \pm 1,21$. A média do número de cigarros consumidos ao dia foi $16,0 \pm 9,3$.

Ocorreu uma diferença significante $(29,2 \pm 1,4 \mathrm{~dB}$ versus $25,2 \pm 2,0 \mathrm{~dB}-\mathrm{t}=6,631 \mathrm{p}<0,0001)$ na média da sensibilidade foveal do olho direito do grupo controle em relação ao mesmo olho do grupo em estudo. Quando comparados os olhos esquerdos dos dois grupos, também ocorreu uma diferença significante $(28,3 \pm$ $1,1 \mathrm{~dB}$ versus $23,8 \pm 3,3 \mathrm{~dB}-\mathrm{t}=4,956 \mathrm{p}<0,0001)$.

Através do gráfico da profundidade do defeito avaliado em decibéis, verificou-se que a maioria dos usuários de tabacoálcool $(64,3 \%)$ apresentava maior número de pontos alterados. Nenhum exame apresentou pontos dentro do expectável. Já no grupo controle todos os exames apresentaram-se com menos de dez pontos alterados, com 8 olhos apresentando de 1 a 3 pontos alterados e o restante sem nenhum ponto alterado (Tabela 1).

Com relação à variação de decibéis, verificou-se que os usuários de tabaco-álcool, apresentaram uma maior freqüência de profundidade do defeito do que o grupo controle (Tabela 2).

A localização do defeito predominante foi a difusa $(54,7 \%)$, seguida das perdas superiores $(14,3 \%)$, temporal $(9,5 \%)$ e as outras regiões em menor intensidade como demonstra a tabela 3.

\section{DISCUSSÃO}

O consumo abusivo do álcool e tabaco causa malefícios em todos os sistemas, inclusive sobre a função visual ${ }^{(3)}$. Alterações na acomodação, midríase e convergência, prejuízo da visão binocular na intoxicação aguda, o fenômeno da inversão binocular profunda, redução da sensibilidade ao contraste e

\begin{tabular}{|c|c|c|c|c|}
\hline \multirow[t]{2}{*}{$\begin{array}{l}\text { Pontos } \\
\text { alterados }\end{array}$} & \multicolumn{2}{|c|}{$\begin{array}{c}\text { Usuários de } \\
\text { tabaco-álcool (olhos) }\end{array}$} & \multicolumn{2}{|c|}{$\begin{array}{c}\text { Controle } \\
\text { (olhos) }\end{array}$} \\
\hline & $n$ & $\%$ & $n$ & $\%$ \\
\hline$<10$ & 15 & 35,7 & 30 & 100 \\
\hline$>10$ & 27 & 64,3 & 0 & 0 \\
\hline \multicolumn{5}{|l|}{$p<0,0001$} \\
\hline
\end{tabular}

\begin{tabular}{|c|c|c|c|c|}
\hline \multirow[t]{2}{*}{$\begin{array}{l}\text { Profundidade } \\
\text { do defeito (dB) }\end{array}$} & \multicolumn{2}{|c|}{$\begin{array}{c}\text { Usuários de } \\
\text { tabaco-álcool (olhos) }\end{array}$} & \multicolumn{2}{|c|}{$\begin{array}{l}\text { Controle } \\
\text { (olhos) }\end{array}$} \\
\hline & $\mathbf{n}$ & $\%$ & $n$ & $\%$ \\
\hline$<10$ & 2 & 4,7 & 30 & 100 \\
\hline$>10$ & 40 & 95,3 & 0 & 0 \\
\hline$p<0,0001$ & & & & \\
\hline
\end{tabular}




\begin{tabular}{|c|c|c|c|}
\hline Local do defeito & $\%$ & Local do defeito & $\%$ \\
\hline Perda difusa & 54,7 & NS & 7,1 \\
\hline $\mathrm{TS}+\mathrm{NS}$ & 14,3 & $\mathrm{NI}+\mathrm{NS}$ & 2,4 \\
\hline $\mathrm{TS}+\mathrm{TI}$ & 9,5 & TS & 2,4 \\
\hline $\mathrm{TI}+\mathrm{NI}+\mathrm{NS}$ & 7,2 & TI & 2,4 \\
\hline
\end{tabular}

discriminação das cores e ambliopia alcoólica já foram descritas associadas ao abuso do álcool ${ }^{(9-15)}$.

A neuropatia óptica tóxica nutricional causa perda da função visual bilateral, lenta e progressiva, levando a escotoma nas áreas central e cecocentral na perimetria convencional acromática $^{(16-17)}$.

Na campimetria azul-amarelo (blue-on-yellow perimetry) ou SWAP são avaliadas as células do sistema parvocelular que transmitem os estímulos visuais responsáveis pela visão de cores que constituem o sistema de células ganglionares azul-amarelo. Neste tipo de teste, a luz amarela é utilizada para causar fadiga dos cones verdes e vermelhos, resultando num isolamento visual dos cones para ondas curtas, onde a luz azul é então detectada ${ }^{(18-19)}$.

A perimetria azul-amarelo, capaz de detectar déficit de campo visual 3 a 5 anos antes da perimetria acromática, tem-se mostrado útil em detectar alterações em pacientes com edema macular diabético e algumas desordens neuro-oftalmológicas ${ }^{(6,8)}$.

Os portadores da neuropatia óptica tóxica nutricional apresentam ao exame, diminuição progressiva da acuidade visual bilateral e palidez temporal do disco óptico ${ }^{(20)}$.

Os resultados deste estudo emprestam suporte ao conceito de que existe uma associação entre o uso crônico de tabaco-álcool e alterações do sistema de células ganglionares azulamarelo, representados pelos números de pontos alterados e a profundidade do defeito campimétrico, sendo a perimetria azul-amarelo 10-2 uma importante ferramenta para detectar as alterações causadas por estas drogas, uma vez que demonstraram alterações nos voluntários com fundoscopia normal e acuidade visual de 1 .

\section{CONCLUSÃO}

Os resultados emprestam suporte ao conceito de que existe uma associação entre o uso crônico de tabaco-álcool e as alterações do sistema de células tipo (ganglionares) cones, sensíveis às cores azuis e amarelas, representadas pelos números de pontos alterados e a profundidade do defeito, sendo indicativo da toxicidade destas drogas para a retina.

\section{ABSTRACT}

Purpose: To evaluate the visual field changes in blue-on-yel- low perimetry (B/Y) strategy 10-2 in alcohol and tobacco smoking consumers. Methods: Forty-two eyes of twenty-one users were studied. Fifteen individuals were used as a control group. All volunteers were males. After normal ophthalmologic examinations, central 10-2 (B/Y) was performed in both eyes. Analysis of the results was performed through the alterations in the depth graph defect and number of altered points. Results: It was found that the majority of the chronic alcohol and tobacco smoking consumers had a greater frequency of alterations in the depth graph defect; 40 eyes (95.3\%), (>10dB), and 27 eyes $(64.3 \%)$ showed a number of altered points, (>10 points), $(\mathrm{p}<0.0001)$. All those who were used as a control group showed alterations in the depth graph defect and number of altered points, but had less than $10 \mathrm{~dB}$ and 10 altered points, respectively. Conclusion: A higher number of abnormal points and depth graph defects and number of altered points were observed in alcohol and tobacco smoking consumers reflecting a higher number of alterations in the cells of the parvocellular system, responsible for color function, by $\mathrm{B} / \mathrm{Y}$ perimetry.

Keywords: Alcoholism; Smoking; Perimetry/methods; Visual fields

\section{REFERÊNCIAS}

1. Bau CHD. Estado atual e perspectivas da genética e epidemiologia do alcoolismo. Ciênc Saúde Coletiva. 2002;7(1):183-90.

2. Del Ben MC, Vilela JAA, Marques JMA. Síndrome de abstinência alcoólica. Medicina (Ribeirão Preto). 1999;32(Supl 1):46-52.

3. Celino AC, Celino M, Toscano J, Diniz JR, Ventura LO. Alterações oculares em pacientes consumidores de álcool. Rev Bras Oftalmol. 2001;60(3):229-32.

4. Strömland K, Pinazo-Durán MD. Ophthalmic involvement in the fetal alcohol syndrome: clinical and animal model studies. Alcohol Alcohol. 2002;37(1):2-8.

5. Yamane R, Dias JFP. Perimetria e campimetria visual manual. In: Yamane R. Semiologia ocular. 2a ed. Rio de Janeiro: Cultura Médica; 2003. p.273-87.

6. Lima FE, Dias JFP. Perimetria computadorizada azul-amarelo e análise da camada de fibras nervosas. In: Dias JFP, Almeida HG. Glaucoma. 2a ed. Rio de Janeiro: Cultura Médica; 2000. p.86-116.

7. Schimiti RB, Costa VP. Perimetria computadorizada Humphrey. In: Yamane R. Semiologia ocular. 2a ed. Rio de Janeiro: Cultura Médica; 2003. p.289-300.

8. Wild JM. Short wavelength automated perimetry. Acta Ophthalmol Scand. 2001;79(6):546-59. Review.

9. Campbell H, Doughty MJ, Heron G, Ackerley RG. Influence of chronic alcohol abuse and ensuing forced abstinence on static subjective accommodation function in humans. Ophthalmic Physiol Opt. 2001;21(3):197-205.

10. Hill JC, Toffolon G. Effect of alcohol on sensory and sensorimotor visual functions. J Stud Alcohol. 1990;51(2):108-13.

11. Watten RG, Lie I. Visual functions and acute ingestion of alcohol. Ophthalmic Physiol Opt. 1996;16(6):460-6.

12. Schneider U, Dietrich DE, Sternemann U, Seeland I, Gielsdorf D, Huber TJ, et al. Reduced binocular depth inversion in patients with alcoholism. Alcohol Alcohol. 1998;33(2):168-72.

13. Donnelly M, Miller RJ. Ingested ethanol and binocular rivalry. Invest Ophthalmol Vis Sci. 1995;36(8):1548-54

14. Braun CM, Richer M. A comparison of functional indexes, derived from screening tests, of chronic alcoholic neurotoxicity in the cerebral cortex, retina and peripheral nervous system. J Stud Alcohol. 1993;54(1):11-6.

15. Kapitany T, Dietzel M, Grünberger J, Frey R, Koppensteiner L, Schleifer G, Marx B. Color vision deficiencies in the course of acute alcohol withdrawal. Biol Psychiatry. 1993;33(6):415-22.

16. Pawlosky RJ, Bacher J, Salem N Jr. Ethanol consumption alters electroretinograms and depletes neural tissues of docosahexaenoic acid in rhesus monkeys: nutritional consequences of a low n-3 fatty acid diet. Alcohol Clin Exp Res. 2001;25(12):1758-65. 
17. Imamura PM. Lesões das vias ópticas. In: Dias JFP, Imamura PM. Campo visual. 2a ed. Rio de Janeiro: Cultura Médica; 2001. p.163-97.

18. Susanna Jr R, Medeiros FA, Barboza WL. Perimetria azul-amarelo. In: Susanna Jr R, Medeiros FA, editores. Perimetria computadorizada: interpretação e discussão de casos. Rio de Janeiro: Cultura Médica; 2001. p.329-53.
19. Costa VP. SWAP (Short - Wavelengh Automated Perimetry) - Perimetria azul amarelo. In: Costa VP. Perimetria computadorizada: um guia prático de interpretação. Rio de Janeiro: Rio Med; 2000. p.141-3.

20. Budenz DL, Siatowski RM. Nonglaucomatous optic nerve disorders. In: Budenz DL. Atlas of visual fields. Philadelphia: Lippincott-Raven; 1997. p.232. 